\title{
Preparation of Biodegradable Polycaprolactone Microcarriers with Doxorubicin Hydrochloride by Ultrasonic-assisted Emulsification Technology
}

\author{
Yeong-Maw Hwang, ${ }^{1}$ Cheng-Tang Pan, ${ }^{1,2 *}$ Yu-Min Lin, ${ }^{1}$ \\ Song-Wei Zeng, ${ }^{1}$ Chung-Kun Yen, ${ }^{1}$ Shao-Yu Wang, ${ }^{1}$ Shiao-Wei Kuo, ${ }^{3}$ \\ Shin-Pon Ju, ${ }^{1}$ Shih-Shin Liang, ${ }^{4}$ and Zong-Hsin Liu ${ }^{5}$ \\ ${ }^{1}$ Department of Mechanical and Electro-Mechanical Engineering, National Sun Yat-sen University, \\ Kaohsiung 80424, Taiwan \\ ${ }^{2}$ Institute of Medical Science and Technology, National Sun Yat-sen University, Kaohsiung 80424, Taiwan \\ ${ }^{3}$ Department of Materials and Optoelectronic Science, National Sun Yat-sen University, Kaohsiung 80424, Taiwan \\ ${ }^{4}$ Department of Biotechnology, Kaohsiung Medical University, Kaohsiung 80424, Taiwan \\ ${ }^{5}$ Metal Industries Research \& Development Centre, Kaohsiung 80424, Taiwan
}

(Received May 31, 2018; accepted October 11, 2018)

Keywords: ultrasonic spray, emulsion-solvent evaporation process, polycaprolactone, biodegradable microcarrier, doxorubicin

Biomedical materials should have biodegradability (enzymatic degradation and hydrolysis) because biodegradable materials in clinical applications show excellent biocompatibility. Polycaprolactone (PCL) is a type of semicrystalline polymer that can be utilized in various organic solvents. Therefore, PCL was made as controlled-release drug carriers for long-term treatment. In this study, we propose a method of preparing biodegradable PCL microcarriers with a uniform and controllable particle size (size range, $\sim 5-100 \mu \mathrm{m}$ ) by emulsion-solvent evaporation with ultrasonic microdroplet spray technology. The emulsion-solvent evaporation was assisted by the ultrasonic spray. Through the standing wave effect on the ultrasonic nozzle, the continuous oil solution of PCL became discontinuous, and the microdroplets of the oil solution were sprayed out directly with high-uniformity particle size. Furthermore, the oil microdroplets of PCL were collected by an aqueous stream in the emulsion process to form the emulsion type of oil in water $(\mathrm{O} / \mathrm{W})$. In addition, the ultrasonic-assisted emulsification technology was adjusted by stirring to enhance the uniformity of particle size. As a result, PCL microdroplets were sprayed into an aqueous solution owing to the low surface tension between the oil and the aqueous solution and the vertical flow field generated by the stirring equipment. The yield rate of PCL microcarriers was improved effectively. Moreover, microcarriers loaded with the cancer drug doxorubicin (DOX), which is water-soluble, were fabricated uniformly with ultrasonic-assisted emulsification technology. The drug loading efficiency (DLE) in this work was $\sim 42.2 \%$, and a microcarrier encapsulation efficiency (EE) of 3.21\% was obtained with a particle size of around 5-20 $\mu \mathrm{m}$.

*Corresponding author: e-mail: pan@mem.nsysu.edu.tw https://doi.org/10.18494/SAM.2019.2093 


\section{Introduction}

In recent years, polymer materials have been extensively applied in biomedical science and technology owing to their excellent biocompatibility and stability in the human body. Some of them even have excellent characteristics, such as nontoxicity and biodegradability, which are important for drug delivery. Moreover, polymer materials have been developed as anticancer drug carriers, which reduce the toxicity of drugs to normal tissues and achieve high therapeutic efficiency. ${ }^{(1-5)}$ Polycaprolactone (PCL) is a semicrystalline polymer that can be utilized in various organic solvents. The characteristics of high degradation temperature and low dissolution temperature of PCL provide good PCL processability. PCL has a repeating structural unit with one polar ester bond and five nonpolar methylene groups, and the ester structure is easily decomposed in nature by microorganisms or enzymes, and the final products are $\mathrm{CO}_{2}$ and $\mathrm{H}_{2} \mathrm{O}$. Owing to the ester hydrolysis of $\mathrm{PCL}$, it is often used as a biodegradable polymer, and its good drug permeability also makes that the drug was released more controllable. $^{(6-9)}$

Several methods of microcarrier fabrication have been described in the literature including precipitation, spraying, phase separation, and emulsion techniques. Emulsion approaches have been commonly used on both the laboratory and industrial scales. ${ }^{(10-12)}$ The control of sphere size and size distribution has several important implications for controlled drug delivery. There is typically an ideal sphere size for release rate and route of administration. There have been several reports of the fabrication of uniform biodegradable polymer microspheres. ${ }^{(13-18)} \mathrm{Kim}$ and Pack ${ }^{(14)}$ have developed a microsphere fabrication technology that combines ultrasonic droplet formation and emulsion-solvent evaporation for generating monodisperse microspheres with precisely controlled size. The technology has been established as a single-step method for producing uniform polymeric microcarriers of controllable size. Monodisperse or precisely defined particle size distributions can be achieved while maintaining the desired polymeric shell thickness. However, monodisperse microsphere fabrication does not have the property of mass production.

To address this situation, in this study, we developed a microsphere fabrication technology that combines emulsion-solvent evaporation with ultrasonic microdroplet atomization technology. There were many applications in the coating field owing to the uniform droplet size. ${ }^{(19-22)}$ A thin liquid film formed on a high-frequency vibrating surface was broken up in a fine uniform spray. The ultrasonic vibration induced surface waves in the liquid film. As the frequency was tuned, very regular square cells can form on the free surface just before reaching the resonance frequency. Then, when resonance is reached, the amplitude grows until droplets are ejected through a crest break-up mechanism. In this study, the liquid film pattern theory was determined mainly using the operating frequency, the surface tension of liquid, the density of liquid, and so forth.

Here, we report the capabilities of two techniques, individually and in combination, for generating uniformed biodegradable microcarriers with precisely controllable size from 5-100 $\mu \mathrm{m}$ diameter. Furthermore, this technology demonstrated to be applicable to doxorubicin (DOX)-loaded microcarrier fabrication. DOX is a common cancer drug that can treat breast cancer, leukemia, lung cancer, liver cancer, and so forth. 


\section{Experimental Methods}

Emulsion-solvent evaporation with ultrasonic microdroplet atomization technology was used to prepare biodegradable polymer microcarriers with a uniform particle size distribution, by analyzing the initial formation of droplets, emulsion-solvent evaporation, particle size shrinkage changes, and anticancer agents for microencapsulation, as shown in schematically Fig. 1.

\subsection{Material}

The PCL $\left(\mathrm{M}_{\mathrm{w}}=10000\right)$ polymer was obtained from Sigma-Aldrich. Polyvinyl alcohol (PVA $88 \%$ hydrolyzed) was also obtained from Sigma-Aldrich. DOX was purchased from Concord Biotech Limited. Ten to $20 \mathrm{ml}$ of 5\% w/w PCL dissolved in ethyl acetate (EA) was prepared as an oil phase solution by emulsion-solvent evaporation. Three hundred milliliters of $5 \% \mathrm{w} / \mathrm{w}$ PVA dissolved in deionized water was prepared as an aqueous-phase solution by emulsionsolvent evaporation.

\subsection{Ultrasonic atomization}

This technology was utilized to accurately control the PCL oil phase solution using an ultrasonic atomizer and sensors to generate a stable quantitative continuous fluid. The nozzle

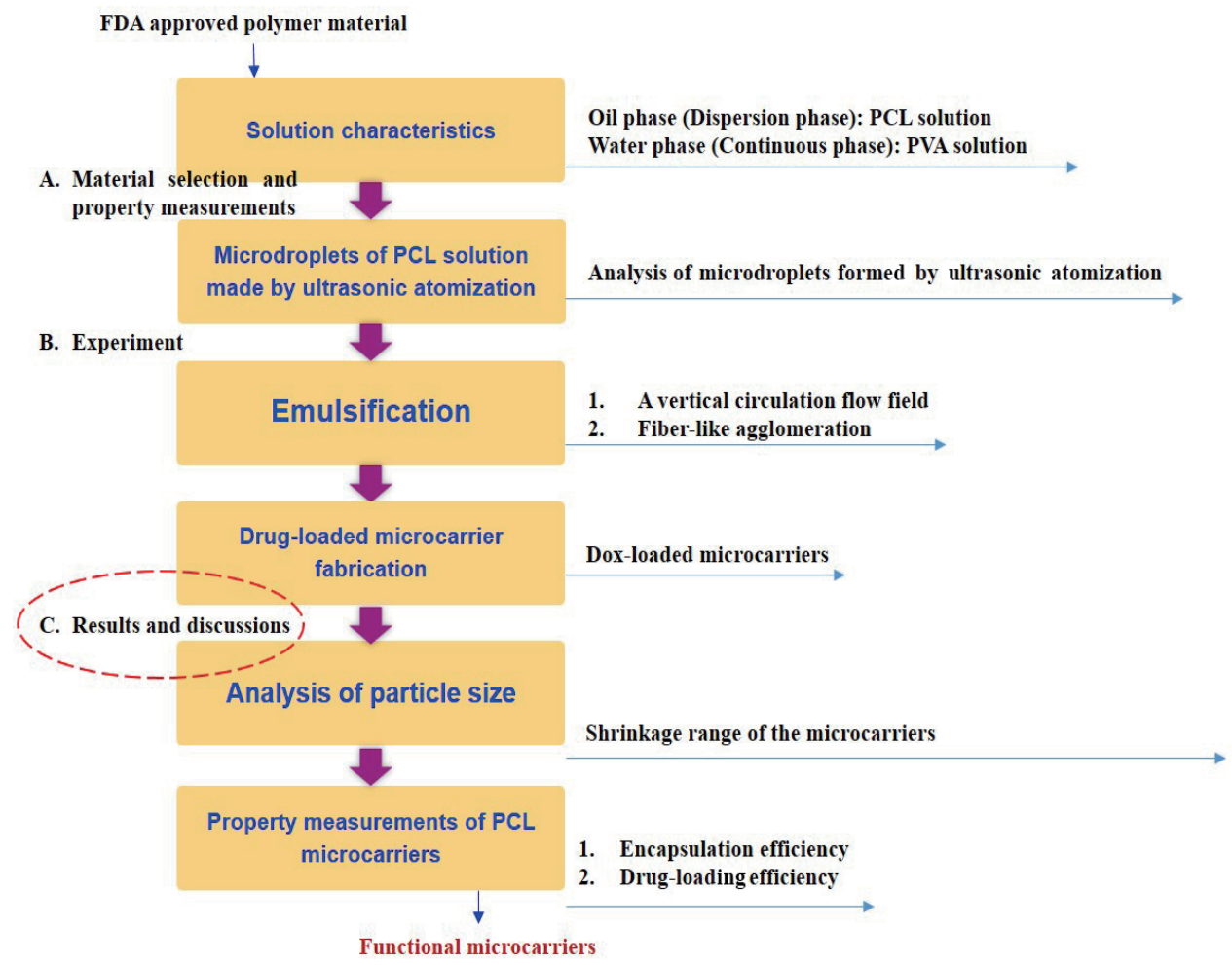

Fig. 1. (Color online) Schematic of experimental process. 
of the atomizer produced $43 \mathrm{kHz}$ of vibration frequency that had a capillary wave effect on the surface of the liquid film. As the amplitude of energy grew and broke through the surface tension of the liquid film, millions of PCL microdroplets were sprayed out directly and gently, ${ }^{(20)}$ as shown in Fig. 2.

\subsection{Emulsion-solvent evaporation}

The 5\% w/w PCL microdroplets were sprayed into a 5\% w/w PVA solution for emulsionsolvent evaporation. The experimental setup is shown in Fig. 3. About $10 \mathrm{ml}$ of the $5 \% \mathrm{w} / \mathrm{w}$ PCL solution was supplied to the ultrasonic atomizer at $0.3 \mathrm{ml} / \mathrm{min}$ using an injection pump. Through the ultrasonic atomizer with $43 \mathrm{kHz}$ frequency, thousands of PCL microdroplets were sprayed into $300 \mathrm{ml}$ of the PVA solution with $200 \mathrm{rpm}$ of stirring. The oil microdroplets of PCL were collected in the PVA solution to form the emulsion type of oil in water $(\mathrm{O} / \mathrm{W})$. In addition, the emulsion-solvent evaporation with ultrasonic microdroplet atomization technology provided a vertical circulation of the flow field of the PVA aqueous solution, as shown in Fig. 4. Through

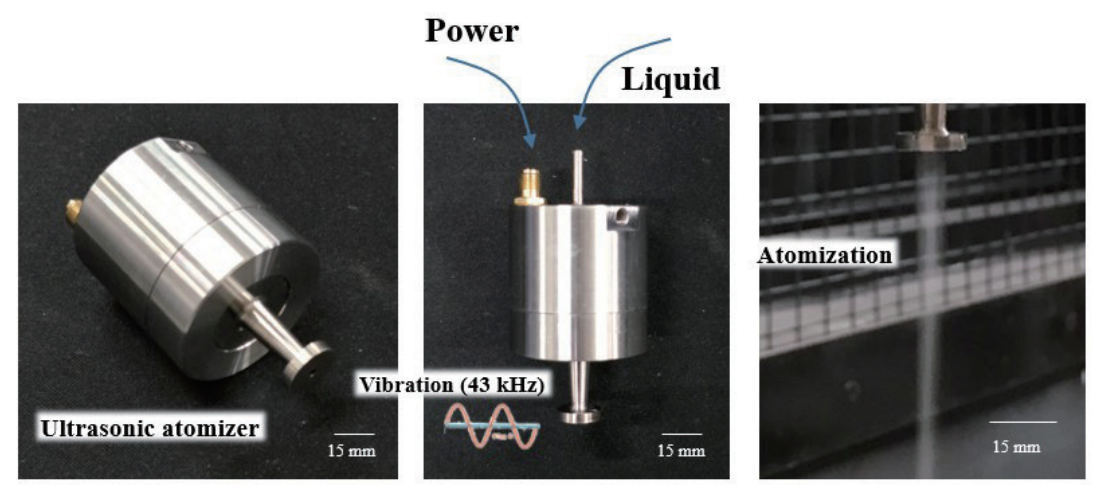

Fig. 2. (Color online) View of ultrasonic atomizer and mechanism of atomization. Energy of ultrasonic-vibrationinduced surface waves in the liquid film. As the frequency reached the resonance frequency, thousands of drops were broken up.

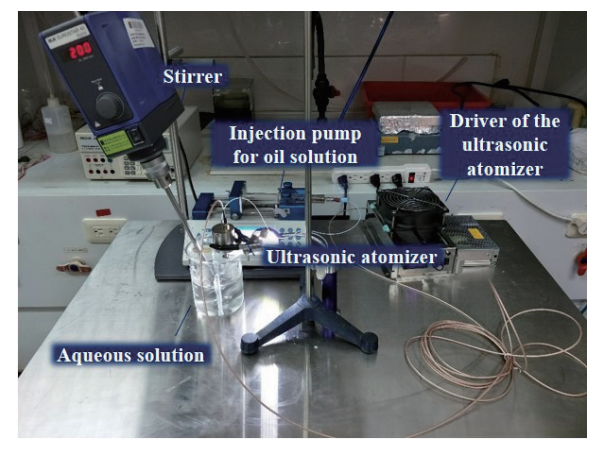

Fig. 3. (Color online) View of equipment, including an ultrasonic atomizer, PCL oil solution, aqueous solution, an injection pump, a stirrer, and a driver of the ultrasonic atomizer.

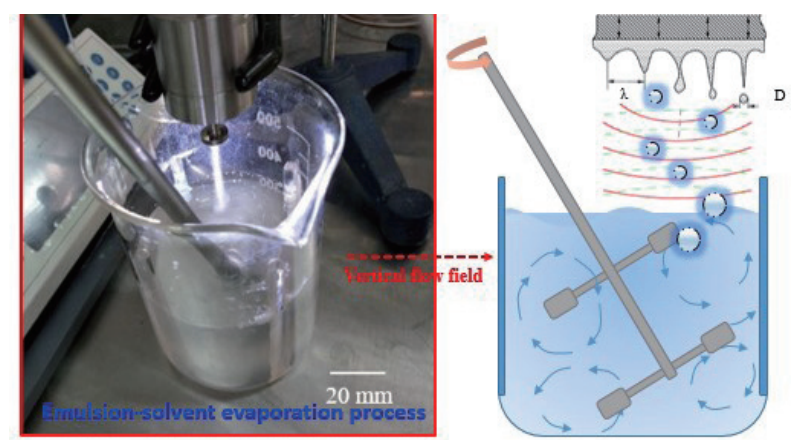

Fig. 4. (Color online) Vertical circulation of flow field to submerge PCL microdroplets into the PVA solution without aggregating. 
the vertical circulation of the flow field, the PCL microdroplets were submerged into the PVA solution without the aggregation of the PCL microdroplets.

\subsection{Particle size distribution measurement}

A laser particle size analyzer (Malvern Spraytec from Kao Yuan University, Taiwn) was employed to analyze the particle size distributions of atomization using the ultrasonic atomizer, as shown in Fig. 5. The laser analyzer with laser beam is used to irradiate the atomization status during the ultrasonic spraying, and the particle size of the microdroplets was observed. The image analysis software image $J$ was used to characterize the particle size distribution of PCL solid microcarriers. Therefore, the formation from microdroplets to solid microcarriers can be observed.

\subsection{Drug-loaded PCL microcarrier preparation}

Fifteen milliliters of 5\% w/w DOX, which is water soluble, was mixed with $5 \% \mathrm{w} / \mathrm{w}$ PCL solution to form the emulsion type of water in oil (W/O) and sprayed using an atomizer, as shown in Fig. 6. About $10 \mathrm{ml}$ of the W/O emulsion solution was supplied to the ultrasonic atomizer at $0.3 \mathrm{ml} / \mathrm{min}$ using an injection pump. Through the ultrasonic atomizer with $43 \mathrm{kHz}$ frequency, thousands of $\mathrm{W} / \mathrm{O}$ emulsion microdroplets were sprayed into $300 \mathrm{ml}$ of PVA solution with stirring at $200 \mathrm{rpm}$. The microdroplets of the $\mathrm{W} / \mathrm{O}$ emulsion solution were collected in the PVA solution and a double emulsion type of water in oil in water $(\mathrm{W} / \mathrm{O} / \mathrm{W})$ was formed.

\subsection{Property measurements of the drug-loaded PCL microcarriers}

A spectrum of DOX was firstly analyzed using a UV-Vis spectrophotometer (Jasco, Model V-770), as shown in Fig. 7. There was a strong absorbance at $230 \mathrm{~nm}$ wavelength. Thus, 230 $\mathrm{nm}$ wavelength was chosen for obtaining the standard calibration curve. Samples of different
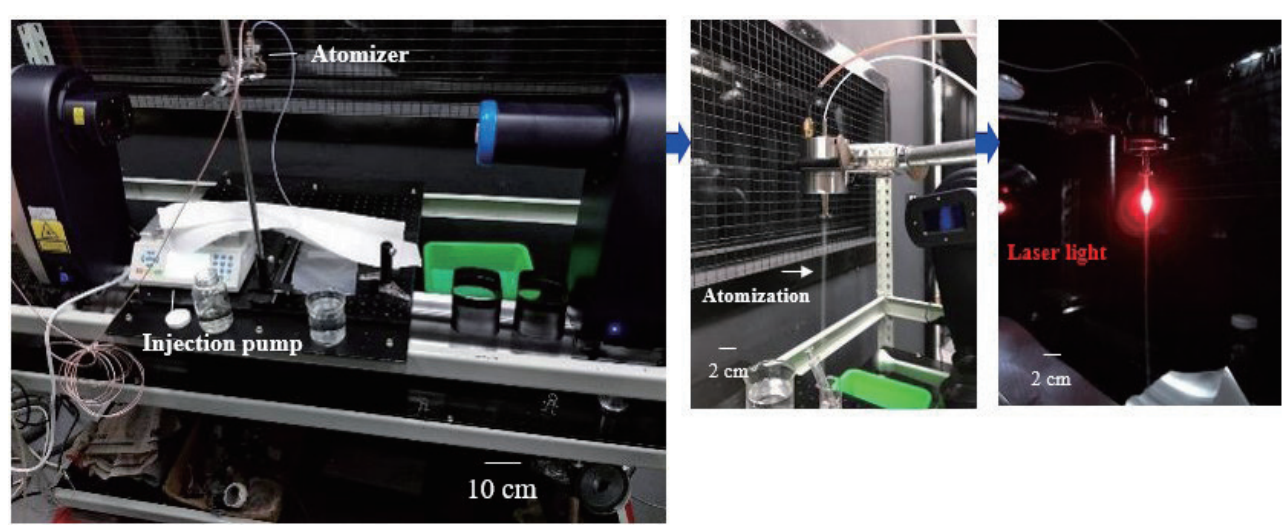

Fig. 5. (Color online) Laser analyzer (Malvern Spraytec) employed to analyze the particle size distribution of the atomization using the ultrasonic atomizer. 


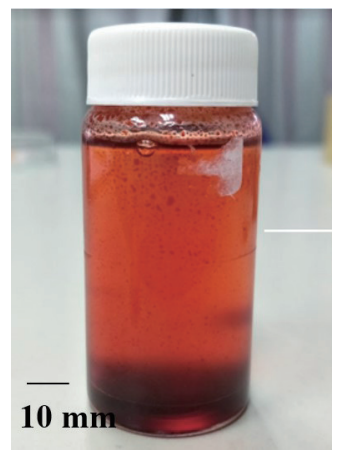

(a)

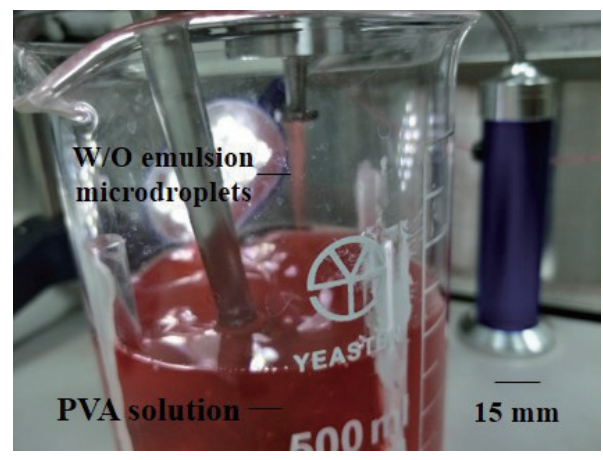

(b)

Fig. 6. (Color online) (a) View of DOX mixed with 5\% w/w PCL solution. (b) View of W/O emulsion microdroplets obtained using the atomizer.

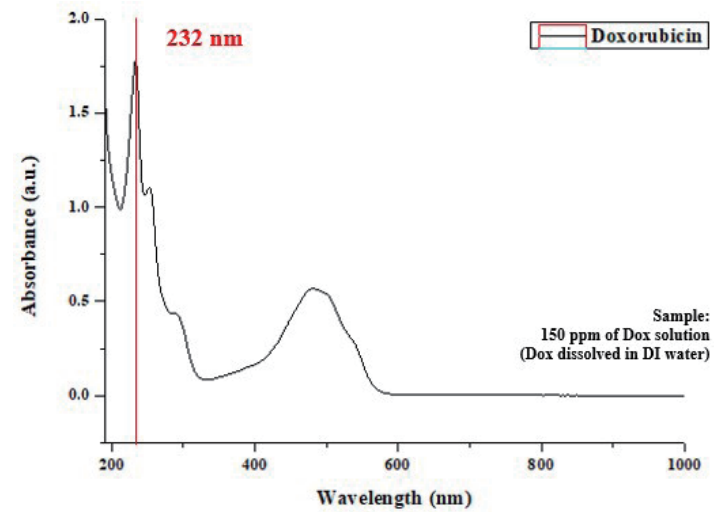

Fig. 7. (Color online) Spectrum of DOX. There was a strong absorbance at $230 \mathrm{~nm}$ wavelength.

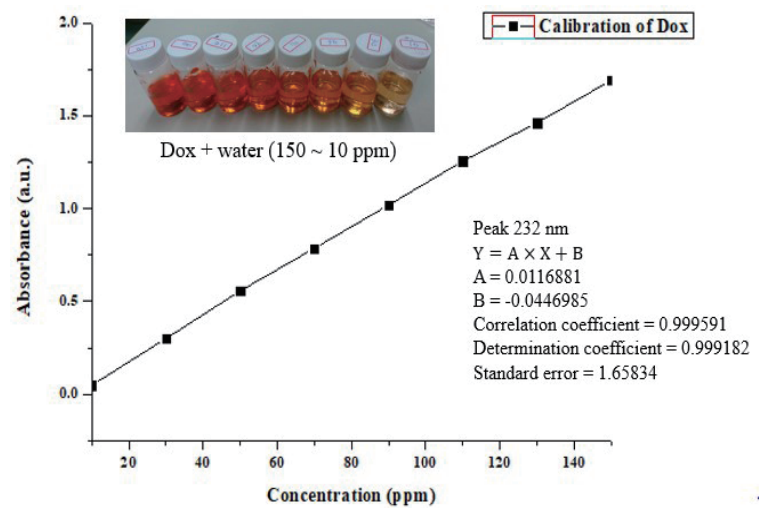

Fig. 8. (Color online) Calibration curve of DOX solution $(0.015-0.4 \mathrm{mM})$.

concentrations $(10-150 \mathrm{ppm})$ of the DOX solution were prepared to obtain the calibration curve, and the result is shown in Fig. 8. The calibration curve is so linear that the concentration of the DOX solution can be calculated on the basis of drug loading efficiency (DLE) and encapsulation efficiency (EE). EE is defined as the percentage of drug weight per unit weight of microcarriers, as shown in Eq. (1). The proportion of the drug in the microcarriers can be observed. DLE is defined as the weight percentage of the drug loaded in all microcarriers per batch of the process, as shown in Eq. (2). How much of the drug is encapsulated or used per process can be observed; in other words, the amount of the drug-contain was obtained by DLE.

$$
E E(\%)=\frac{\text { Total weight of drug in microcarriers }}{\text { Total weight of microcarrers }}
$$

$$
D L E(\%)=\frac{\text { Total weight of drug in all microcarriers by one process }}{\text { Total weight of drug introduced in the process }}
$$




\section{Results and Discussion}

\subsection{Emulsion-solvent evaporation with ultrasonic microdroplet spray technology}

The main process for the PCL microspheres is schematically shown in Fig. 9. Lipophilic fragments of PVA attached onto the surface of the oil droplets to achieve three-dimensional stability and make the emulsion droplets stable and evenly dispersed. As the solvent EA of PCL microdroplets diffused into the aqueous solution or evaporated into the air, the PCL microdroplets formed semicured microcarriers gradually from the interface to the core. After the solvent was completely removed from the PCL microdroplets, the PCL microcarriers were obtained, as shown in Fig. 10.

In traditional emulsification, the particle sizes of microcarriers are determined by the high sheer force (stirring speed was more than $1000 \mathrm{rpm}$ ) of the PVA solution. Compared with the traditional method, the emulsion-solvent evaporation with ultrasonic microdroplet spray technology was employed with the ultrasonic atomizer to produce thousands of microdroplets and determine firstly their size distributions without considering the low shear force (stirring speed was $\sim 200 \mathrm{rpm}$ ) of the PVA solution. However, the kinetic energy of this gentle and directional atomization was too low to break the surface tension of the PVA solution, and the solvent was used to change the surface tension of PVA solution to allow PCL microdroplets to be able to submerge into the PVA solution. Many PCL microdroplets had a fiberlike agglomeration owing to premature precipitation as the solvent EA has a high water solubility (86 g EA/1 $\mathrm{L} \mathrm{H}_{2} \mathrm{O}$ ), as shown in Fig. 11. To avoid this phenomenon, the inclined stirring rod and inner baffle plate design of the PVA solution beaker were used to produce a stable low vertical shearing effect, thereby providing a more stable emulsification environment for the emulsion droplets and effectively submerging PCL microdroplets into the PVA solution, as shown in Fig. 12.

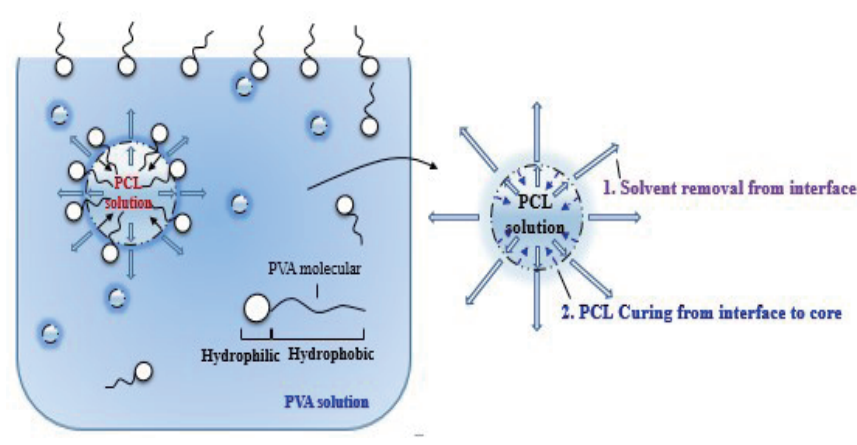

Fig. 9. (Color online) Emulsion-solvent evaporation process.

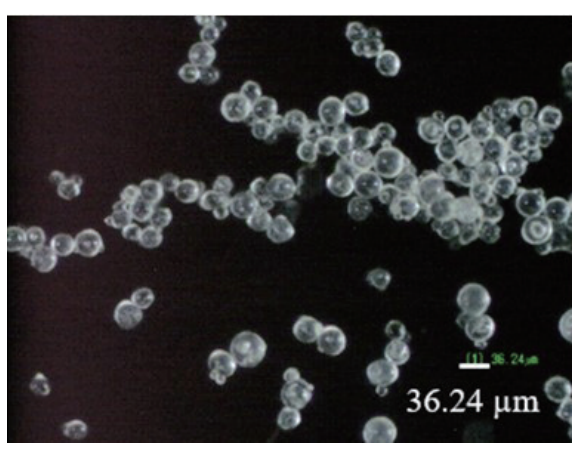

Fig. 10. (Color online) View of pure PCL microcarriers. 

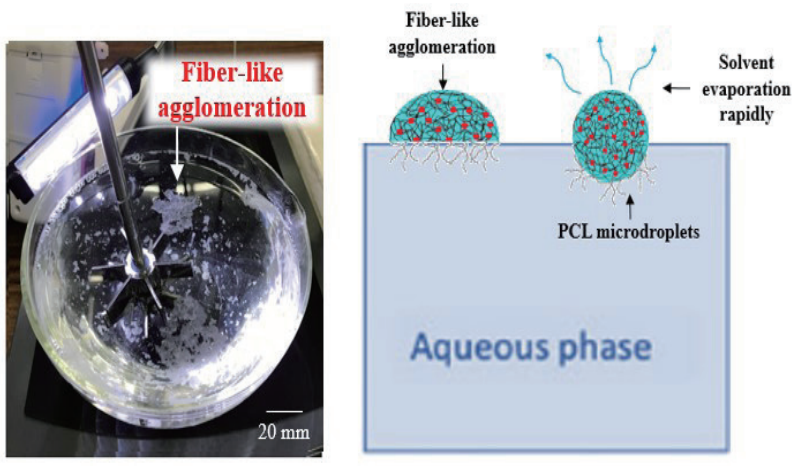

Fig. 11. (Color online) Premature precipitation of PCL microdroplets and fiberlike agglomeration phenomenon.

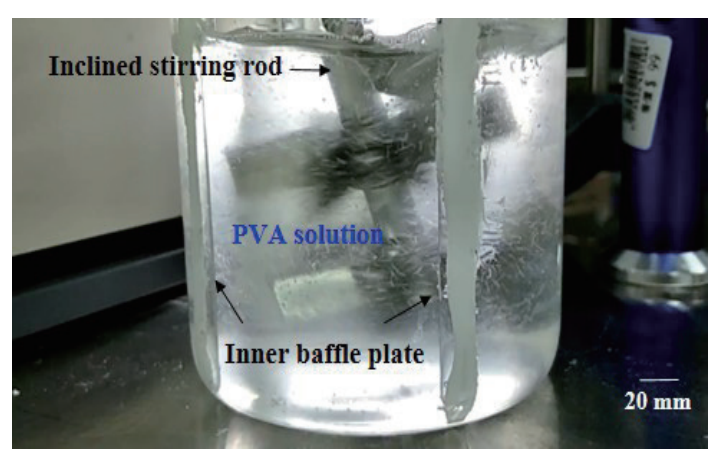

Fig. 12. (Color online) Inclined stirring rod and inner baffle plate design of the PVA solution beaker.

\subsection{Particle size distribution measurement}

Under a stable rotational speed, the PCL microdroplets were solidified as the solvent diffused into the PVA solution, and the PCL droplets solidified from the interface to the core. By the emulsion-solvent evaporation with ultrasonic microdroplet spray technology, the size of the PCL microdroplets was mainly determined by the ultrasonic atomization (particle sizes $\sim 15-60 \mu \mathrm{m}$ ). From the results obtained with the laser particle size analyzer, the median particle size (Dv 50) after the atomization of the $5 \% \mathrm{w} / \mathrm{w}$ PCL solution was $\sim 32 \mu \mathrm{m}$. From the image $\mathrm{J}$ results, the median particle size (Dv 50) of the solidified carriers was $\sim 8 \mu \mathrm{m}$. Therefore, the analysis of particle size showed that the particle sizes of the microdroplets that form solidified carriers shrank by about $\sim 40 \%$, as shown in Fig. 13 .

\subsection{Drug-loaded microcarriers}

DOX-loaded microcarriers were produced, as shown in Fig. 14. The DLE in this process was $\sim 42.2 \%$, and the EE of these microcarriers was $\sim 3.21 \%$, as calculated using Eqs. (1) and (2). Through the technology, DOX was encapsulated into the PCL microcarriers by forming multiple emulsions of water-oil-water $\left(\mathrm{W}_{1} / \mathrm{O} / \mathrm{W}_{2}\right)$. However, the DOX solution was not evenly disperesed in the PCL solution, and the mixture with complex solution properties (such as density, surface tension, and vicosity) showed unstable atomization causing uncontrollable particle size. The stratification system can be divided into two parts. The upper layer solution has a relatively low density of the PCL solution $\left(\rho, \sim 0.897 \mathrm{~g} / \mathrm{cm}^{3}\right)$ with a low density of the DOX solution. The lower layer solution has a high density of the DOX solution $\left(\rho, \sim 1 \mathrm{~g} / \mathrm{cm}^{3}\right)$ with a low density of the PCL solution. Since DOX diffused into the PVA solution similarly to the solvent EA, the W/O emulsion with the higher density of the DOX solution did not indicate that a higher EE of the PCL microcarriers would be obtained. There are many factors that would affect EE, such as temperature, solution concentration, and process time, which will be considered in a future study. The rate of solvent evaporation in emulsification could be a 


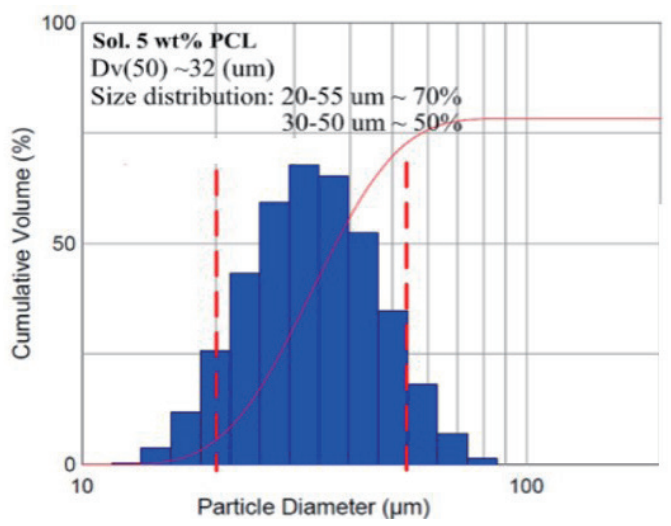

(a)

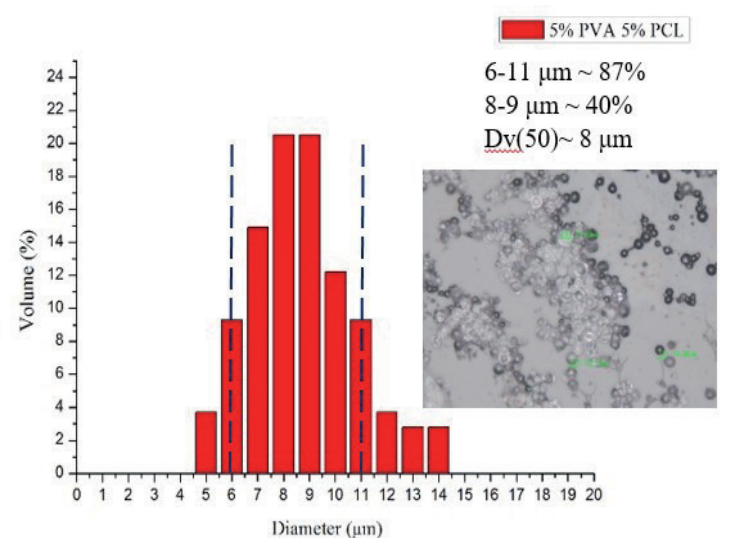

(b)

Fig. 13. (Color online) Analysis of particle size: (a) microdroplets after atomization and (b) solidified carriers.

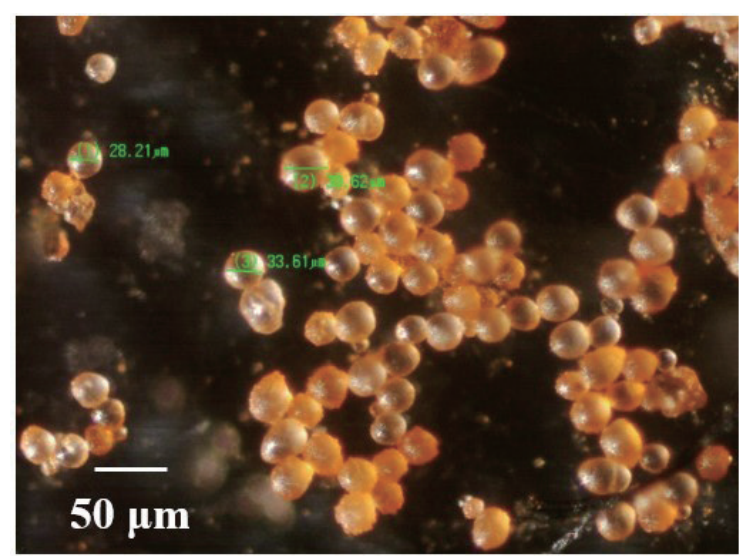

Fig. 14. (Color online) View of DOX-loaded PCL microcarriers.

significant factor for obtaining a high EE of microcarriers. The rate of solvent evaporation determines how completely and rapidly the drug-loaded microcarriers form.

\section{Conclusion}

Biodegradable PCL microcarriers with uniform and controllable particle size (size range, $\sim 5-100 \mu \mathrm{m})$ were fabricated successfully by emulsion-solvent evaporation with ultrasonic microdroplet atomization technology. This innovative method, which combined emulsionsolvent evaporation with ultrasonic microdroplet atomization technology, not only produced a narrow particle size distribution of microcarriers, but also provided controllable particle size, high yield, short process time, and simple operation. With this technology, an ultrasonic atomizer is employed to produce stable PCL microdroplets, and highly uniform microcarrier formation control is achieved through the low-shear-force effect and the vertical circulation of the flow field during the emulsion-solvent evaporation. This curing environment of PCL microcarriers made it possible to control effectively the particle size of microdroplets to form 
solidified carriers (shrinkage range, $\sim 40 \%$ ). Furthermore, this technology demonstrated the fabrication of microcarriers loaded with DOX. The DLE in this process was $\sim 42.2 \%$, and the $\mathrm{EE}$ of these microcarriers was $\sim 3.21 \%$. In the future, biodegradable drug-loaded carriers will be more important for long-term cancer treatment. The fabrication of these carriers with uniform and controllable particle size will be more valuable.

\section{References}

1 A. Ito, M. Shinkai, H. Honda, and T. Kobayashi: J. Biosci. Bioeng. 100 (2005) 1.

2 H. Otsuka, Y. Nagasaki, and K. Kataoka: Adv. Drug Delivery Rev. 55 (2003) 403.

3 V. P. Torchilin: Adv. Drug Delivery Rev. 58 (2006) 1532.

4 I. Brigger, C. Dubernet, and P. Couvreur: Adv. Drug Delivery Rev. 54 (2002) 631.

5 W. Zhang and C. Gao: Chin. Sci. Bull. 60 (2015) 1973.

6 M. A. Woodruff and D. W. Hutmacher: Prog. Polym. Sci. 35 (2010) 1217.

7 Y. Ikada and H. Tsuji: Macromol. Rapid Commun. 21 (2000) 117.

8 C. M. Agrawal and R. B. Ray: J. Biomed. Mater. Res. 55 (2001) 141.

9 J.-M. Idée and B. Guiu: Crit. Rev. Oncol. Hemat. 88 (2013) 530.

10 S. Freitas, H. P. Merkle, and B. Gander: J. Controlled Release 102 (2005) 313.

11 M. Li, O. Rouaud, and D. Poncelet: Int. J. Pharm. 363 (2008) 2008.

12 F. Dahmoune, F. Rezgui, and C. G'Sell: Mater. Sci. Eng. C 58 (2016) 412.

13 C. Berkland, K. K. Kim, and D. W. Pack: J. Controlled Release 73 (2001) 59.

14 K. K. Kim and D. W. Pack: BioMEMS and Biomedical Nanotechnology (2006) 19.

15 R. Liu, G. H. Ma, F. T. Meng, and Z. G. Su: J. Controlled Release 103 (2005) 31.

16 Y. C. Lin, H. S. Lin, Z. H. Hong, and Z. H. Liu: Microsyst. Technol. 23 (2017) 525.

17 B. Liu, S. Sun, M. Zhang, L. Ren, and H. Zhang: Colloids Surf., A 484 (2015) 81.

18 Y. Sasaki, N. Konishi, M. Kasuya, M. Kohri, T. Taniguchi, and K. Kishikawa: Colloids Surf., A 482 (2015) 68.

19 S. Sajjadi and Y. Chen: Eur. Polym. J. 69 (2015) 364.

20 Y. M. Han, B. H. Son, S. M. Hong, and S. B. Choi: Smart Mater. Struct. 20 (2011) 107001.

21 J. Riemer: Ultrasonic Spray Coating of Nanoparticles (2011) 26.

22 R. Engle: J. Fuel Cell Sci. Technol. 9 (2012) 637. 